\title{
Tumores bilaterales de testículo
}

\author{
Diz Rodríguez R, Vírseda Chamorro M, Arance Gil I, Sáenz Benito D, Alpuente Román C, \\ Paños Lozano P.
}

Servicio de Urología. Hospital Central de la Defensa. Madrid.

Actas Urol Esp 2007;31(3):250-252

\section{RESUMEN}

TUMORES BILATERALES DE TESTÍCULO

Objetivos: Estudiar la incidencia y características de los tumores bilaterales de testículo.

Material y métodos: Se realizó un estudio retrospectivo sobre una base de datos de tumores 98 testiculares tratados en nuestro servicio entre los años 1979 y 2004.

Resultados. Se registraron 4 casos de tumores bilaterales (un 4,1\%) en la serie. El intervalo de aparición del segundo tumor osciló entre 14 meses y 4 años y medio (siendo la mediana de 47 meses). En tres casos el tumor inicial fue un Carcinoma embrionario y en uno un tumor de Leydig. En dos casos el segundo tumor fue del mismo tipo histológico (Ca embrionario y tumor de Leydig), mientras que en los otros dos casos de Ca embrionario, el segundo tumor fue un seminoma y un teratocarcinoma.

Respecto a la histología del primer tumor, se observó que sólo 3 de los 27 Ca embrionarios (11\%) de nuestra serie, experimentaron una segunda neoplasia frente a 1 de los 2 tumores de Leydig (50\%).

Conclusiones. La incidencia de tumores bilaterales en nuestra serie fue del $4,1 \%$, en nuestra serie el riesgo de aparición de un segundo tumor parece ser mas elevado en pacientes con tumores de Leydig aunque el pequeño número de caso no son permite extraer conclusiones significativas.

Palabras clave: Tumor germinal de testículo bilateral. Histología. Tumor de células de Leydig.

\section{ABSTRACT}

\section{BILATERAL TESTICULAR TUMOURS}

Objectives: To study the incidence and characteristic of the bilateral tumours of testicle.

Material and methods. It was carried out a retrospective study on a database of testiculars tumours 98 tried in our service among the years 1979 and 2004.

Results. We registered 4 cases of bilateral tumours $(4,1 \%)$ in the series. The interval of appearance of the second tumor oscillated between 14 months and 4 and a half years (being the medium of 47 months). In three cases the initial tumour was an embryonic Carcinoma and in one a tumour of Lydia. In two cases the second tumour was of the same type histological (embryonic Ca and tumour of Leydig), while in the other two cases of embryonic $\mathrm{Ca}$, the second tumour was a seminoma and a teratocarcinoma.

Regarding the histology of the first tumour, it was observed that only 3 of the 27 embryonic Ca (11\%) of our series, they experienced a second neoplasia in front of 1 of the 2 tumours of Leydig (50\%).

Conclusions. The incidence of bilateral tumours in our series was of $4,1 \%$. In our series the risk of the second tumour seems to be higher in patients with Leyding tumours, therefore the lesser number of tumours do not allow us to know significantly conclusions.

Keywords: Germinal bilateral tumour of testicle. Histology. Leydig's tumour.

$\mathrm{L}$ os tumores testiculares representan alrededor del 1\% de los tumores del varón ${ }^{1}$. El 95\% de estas neoplasias corresponde a tumores germinales cuya máxima incidencia tiene lugar entre la segun- da y tercera década de la vida adulta ${ }^{2}$. La aparición de una segunda neoplasia en el testículo contralareral es poco frecuente, estimándose en alrededor del 3\% del total de tumores testiculares ${ }^{3}$. 
El objetivo de nuestro trabajo es investigar las características y tiempo de evolución de los casos en los que se diagnosticó un segundo tumor en el testículo contralateral.

\section{MATERIAL Y MÉTODOS}

Se realizó un estudio retrospectivo en una cohorte de 98 varones diagnosticados en nuestro Servicio de neoplasia testicular entre 1979 y 2004. Se recogieron datos referentes a la edad de los pacientes, testículo afectado, tipo y estadio histológico tumoral, tratamiento efectuado, y aparición de una segunda neoplasia en el testículo contralateral. El tiempo de seguimiento medio de los pacientes fue de 63 meses (desviación típica de 82 meses), los que indica que el tiempo de seguimiento no se ajusta a una distribución normal (mediana de 41 meses, amplitud intercuartil 85,37 meses).

El tipo histológico se estableció de acuerdo con la clasificación del Mostofi ${ }^{4,5}$. La extensión tumoral se evaluó mediante la realización de radiografía de tórax, y TAC abdominal y torácico. El estadio tumoral se determinó de acuerdo con la clasificación del hospital Royal Madsen ${ }^{6}$.

El análisis estadístico se realizó mediante el test de comparación de medias de la t de Student para variables niméricas, y el test exacto de Fisher para variables dicotómicas.

\section{RESULTADOS}

En nuestra serie se encontraron cuatro casos de tumor testicular contralateral, lo que correspondió a un $4,1 \%$ del total de neoplasias testiculares revisadas. El intervalo de aparición del segundo tumor osciló entre 14 meses y 4 años y medio (siendo la mediana de 47 meses).

La edad media de los pacientes con una segunda neoplasia testicular fue de 26,2 años (desviación típica 3,0 años). La edad media de los pacientes que no presentaron una segunda neoplasia testicular fue de 28,9 años (desviación típica 14,2 años). Las diferencias no fueron significativas.

El testículo donde apareció el primer tumor fue el izquierdo en tres casos (75\%), y el derecho en uno (25\%). En los pacientes en los que no apareció un segundo tumor, el tumor apareció en el testículo izquierdo en 30 casos (37\%) y en el derecho en 50 casos (63\%). Las diferencias no fueron significativas.
El tipo de tumor primario y segundario en los pacientes con segunda neoplasia testicular se muestran en la Tabla 1. La comparación de la histología tumoral con el porcentaje de tumores contralaterales se muestra en la Tabla 2 . El porcentaje de pacientes con tumor de Leydig y recidiva contralatreral tendió hacia la significación estadística $(\mathrm{p}=0,061)$.

Tabla 1

Tipo de neoplasia en los tumores testiculares recidivantes.

\begin{tabular}{lll}
\hline No paciente & Primer tumor & Segundo tumor \\
\hline 1 & Ca embrionario & Ca embrionario \\
2 & Ca embrionario & Teratocarcinoma \\
3 & Ca embrionario & Seminoma \\
4 & Tm de Leydig & Tm de Leydig \\
\hline
\end{tabular}

Tabla 2

Comparación entre el tipo histológico y la recidiva contralateral de la neoplasia.

\begin{tabular}{lcc}
\hline $\begin{array}{l}\text { Tipo histológico } \\
\text { primario }\end{array}$ & No de casos & $\begin{array}{c}\text { No de casos con tumor } \\
\text { contralateral* }\end{array}$ \\
\hline Seminomas & 27 & $0(0 \%)$ \\
Ca embrionario & 26 & $3(11 \%)$ \\
Tm de Leydig & 2 & $1(50 \%)$ \\
Otros tipos & 43 & $0(0 \%)$ \\
Total & 98 & $4(4 \%)$ \\
\hline
\end{tabular}

* Entre paréntesis porcentaje de pacientes con un segundo tumor contralateral.

La comparación entre el estadio tumoral de los tumores con recidiva contralateral y sin recidiva contralateral se muestra en la Tabla 3. Las diferencias no fueron significativas.

Tabla 3

Comparación entre el estadio tumoral y la recidiva contralateral de la neoplasia.

\begin{tabular}{lcc}
\hline $\begin{array}{l}\text { Estadio } \\
\text { tumoral }\end{array}$ & No de casos & $\begin{array}{c}\text { No de casos con tumor } \\
\text { contralateral* }\end{array}$ \\
\hline I & 33 & $1(3 \%)$ \\
II & 17 & $2(12 \%)$ \\
III & 5 & $1(20 \%)$ \\
IV & 1 & $0(0 \%)$ \\
No registrado & 42 & 0 \\
Total & 56 & $4(7 \%)$ \\
\hline
\end{tabular}

*Entre paréntesis porcentaje de pacientes con un segundo tumor contralateral. 
El tratamiento consistió en orquiectomía inicial y contralateral en todos los casos. Inicialmente se administró quimioterapia tipo BEP en los tres casos de Ca embrionario y sólo orquiectomía en el caso del tumor del Leydig. Tras la recidiva tumoral sólo se administró quimioterapia en el caso del paciente con teratocarcinoma. El tiempo de seguimiento de los pacientes con la según da neoplasia fue de 8,37 meses (desviación típica: 6,5 meses). No se observó recidiva en ninguno de los casos.

\section{DISCUSIÓN}

La prevalencia de tumores testiculares bilaterales en nuestra serie fue del 4,1\%. En otras series esta prevalencia oscila entre el 2 y el 5\% de los tumores testiculares ${ }^{7}$. En la mayoría de los casos el desarrollo del segundo tumor ocurre después de la aparición del primer tumor (metacrónico). Ondrus et $\mathrm{al}^{8}$ refieren únicamente dos casos de tumor bilateral sincrónico en los doce casos de tumores testiculares bilaterales, siendo el intervalo de aparición de los tumores metacrónicos entre tres y trece años. En nuestra serie los cuatro tumores fueron metacrónicos con un intervalo de aparición entre catorce meses y cuatro años y medio.

Generalmente el segundo tumor tiene la misma estirpe histológica que el primero, siendo inusual que sea de otro tipo histológico. Dieckmann and Loy $^{7}$ describen la aparición excepcional de un tumor de Leydig contralateral siete años después de la extirpación del otro testículo por un tumor testicular mixto (carcinoma embrionario, teratoma y tumor de Yolk Sac). En nuestra serie encontramos dos casos de diferente estirpe tumoral entre el primer y segundo tumor: de carcinoma a seminoma y a teratocarcinoma.

Entre los factores de riesgo para la aparición de un segundo tumor en el otro testículo se ha descrito la presencia de un carcinoma in situ contralateral. Christensen et $\mathrm{al}^{9}$ consideran que hasta un 5\% de los pacientes con neoplasia testicular tendrían un carcinoma in situ contralateral, que progresaría hasta un carcinoma invasivo en la mitad de los casos. Los tumores de Leydig son neoplasias que afectan a las células testiculares encargadas de segregar los andrógenos. Su prevalencia es baja (alrededor del 10\% de los tumores testiculares) ${ }^{10}$. Se han descrito varios casos de neoplasia metacrónica en el testículo contralateral siempre de la misma estirpe tumoral ${ }^{10-12}$. Este tipo de tumor tiene un mayor riesgo de presentar una segunda neoplasia en el testículo contralateral, estimándose entre el 5 a 10\% de los casos (frente al 1,5\% del resto de tumores) ${ }^{13}$ En nuestra serie se encontraron dos casos de tumor de células de Leydig, de los que uno presentó una segunda neoplasia contralateral a los catorce meses. Sin embargo, debido el pequeño número de casos no podemos extraer conclusiones significativas.

\section{REFERENCIAS}

1. Schottenfield D, Warshauwe ME, Sherlock S, Zauber AG, Leyder M, Payne R. The epidemiology of testicular cancer in young adults. Am J Epidemiol. 1980;112(2):232-246.

2. Richie JP. Neoplasm of the testis. In Walsh, Retik, Stamey and Vaughan editors. Campbell's Urology $7^{\text {th }}$ ed. Philadelphia, Wb Saunder Co. 1997.2411-2452.

3. Ohyama C, Kyan A, Satoh M, Saito S, Nishimura Y, Imai Y, et al. Bilateral testicular tumors: a report of nine cases with long-term follow-up. Int J Urol. 2002;9(3):173-177.

4. Mostofi FK, Sobin LH. International histological clasification of tumor of the teste. Geneve. WHO. 1977.

5. Mostofi FK, Sesterhenn IA. Histological typing of the testis tumors. WHO International Histological Clasification Tumors $2^{\text {nd }}$ Edition . Berlin. Springer-Verlag. 1998.

6. Medical Research Council Working Party on Testiculars Tumours Prognostic factors in advanced non-seminomatous germ-cell testicular tumours: results of multicentre study. Report from the Medical Research Council Working Party on Testicular Tumours. Lancet. 1985;1(8419):8-11.

7. Dieckmann KP, Loy V. Metachronous germ cell and Leydig cell tumors of the testis. Do testicular germ cell tumors and Leydig cell tumors share common etiologic factors? Cancer. 1993;72(4): 1305-1307.

8. Ondrus D, Matoska J, Hornak M. Bilateral germ cell tumors of the testis. Neoplasma. 1993;40(5):329-332.

9. Christensen TB, Daugaard G, Geertsen PF, von der Maase H. Effect of chemotherapy on carcinoma in situ of the testis. Ann Oncol. 1998;9(6):657-660.

10. Sugimura J, Suzuki Y, Tamura G, Funaki H, Fujioka T, Satodate R. Metachronous development of malignant Leydig cell tumor. Hum Pathol. 1997;28(11):1318-1320.

11. Pereira Arias JG, Ateca Diaz-Obregon R, Ullate Jaime V, Gutierrez Diez JM, Ramirez Rodriguez MM, Etxezarraga Zuluaga MC, et al. Tumor de Leydig contralateral metacrónico del testiculio: tratamiento conservador. Actas Urol Esp. 2001;25(2):133-138.

12. Praz V, Strebel R, Regusci S, Sarazin D, Hauri D. Metachronous bilateral leydig cell tumor in a male adult aged 20. Urol Int. 2004;73(4):370-373.

13. Colls BM, Harvey VJ, Skelton L, Thompson PI, Frampton CM. Bilateral germ cell testicular tumors in New Zealand: experience in Auckland and Christchurch 1978-1994. J Clin Oncol. 1996; 14(7):2061-2065.

Correspondencia autor: Dr. M. Vírseda Chamorro

Servicio de Urología. Hospital Central de la Defensa.

Glorieta del Ejército, s/n. 28047 Madrid. Tel.: 914228701

E-mail autor: bgmeli@terra.es

Información artículo: Original - Cáncer renal

Trabajo recibido: septiembre 2006

Trabajo aceptado: enero 2007 